\title{
PHOTOELECTRON IMAGING IN CELL BIOLOGY
}

\section{O. Hayes Griffith}

Institute of Molecular Biology and Department of Chemistry, University of Oregon, Eugene, Oregon 97403

\section{Gertrude F. Rempfer}

Department of Physics, Portland State University, Portland, Oregon 97207

\section{CONTENTS}

Perspectives and Overview..

Historical.

The Photoelectron Microscope.

Theory and Strategy of Cell Surface Imaging.

Photoelectron Images of Eucaryotic Cells.

\section{Perspectives and Overview}

Recent progress in the study of the biochemical functions of hormone receptors, growth factor receptors, receptor-mediated endocytosis, the immune response, and tumor-associated antigens has increased efforts to map the surfaces of eucaryotic cells. The complexity of the problem is inherently great because the plasma membrane is a thin and very delicate structure and it constitutes only a small fraction of the total quantity of cellular membrane. Furthermore, specific receptors constitute a relatively small fraction of the plasma membrane constituents, which include a great diversity of different receptors, lipids, and other functional proteins. The relative locations of surface receptors and any relationship to cytoskeletal elements within the cell are largely unknown. To approach this problem requires a combination of available microscopic techniques, monoclonal antibody technology, and biochemistry. Figure 1 illustrates several electron 
TECHNIQUES FOR IMAGING CELL SURFACES

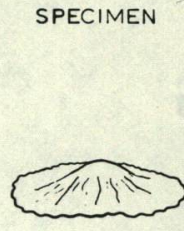

a

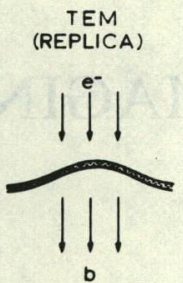

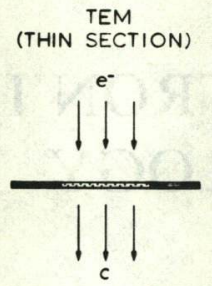

SEM

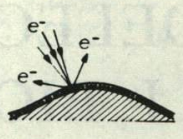

d
PEM

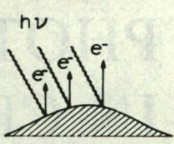

e

Figure 1 Comparison of some of the ways of visualizing biological surfaces. (a) Sketch of a cell surface, $(b-d)$ the standard electron optical methods, and $(e)$ photoelectron imaging of the uncoated cell surface.

microscope techniques for imaging cell surfaces. The established methods include transmission electron microscopy (TEM) of replicas, serial sections, or whole mounts, and scanning electron microscopy (SEM) of whole mounts coated with a conducting layer. Figure 1 also includes a sketch illustrating a relatively new entry in the field, photoelectron microscopy (PEM). Our purpose here is to describe the emerging technique of photoelectron imaging in cell biology and then to discuss in general the factors that need to be considered when imaging biological structures. ${ }^{1}$

\section{Historical}

Most of us were introduced as freshmen to the photoelectric effect in terms of its impact on the quantum theory, through a diagram similar to Figure 2. When light of sufficiently short wavelength strikes the cathode, photoelectrons are produced creating a current in the circuit. Two characteristics of photoelectron emission were puzzling from the point of view of classical physics: (a) a threshold frequency of the incident light is required to produce photoelectrons, and $(b)$ the maximum kinetic energy of the photoelectrons is a linear function of the light frequency. Einstein in 1905 proposed that light itself was quantized (19). That is, that light is transmitted as packets, or quanta, of energy $(E)$ related to the frequency $(v)$ by the simple but enormously powerful equation

$$
E=h v
$$

where $h$ is Planck's constant. This led to Einstein's photoelectric equation

$$
(1 / 2) m v^{2}=h v-\phi
$$

${ }^{1}$ A preliminary discussion of some of these factors was presented in conference proceedings (14). 
where $(1 / 2) m v^{2}$ is the kinetic energy of the electron, and $\phi$ is the energy required to release the electron from the sample surface (also called the work function).

The kinds of information available from the emitted electrons of Figure 2 include the photoelectron yield, the kinetic energy distribution, the angles of emission, and, most importantly for our purposes, the positions at which the electrons leave the surface. The history of the photoelectric effect has followed several separate paths. The simple circuit of Figure 2 has evolved into the modern photocathode, the detector of light levels in instruments such as optical spectrometers commonly found in biochemistry laboratories. A separate development involved the introduction of energy analyzers to sort out the kinetic energy distributions, in analogy with the introduction of monochromators into light beams. This has led to modern photoelectron spectroscopy, which is generally divided into two fields: Xray photoelectron spectroscopy (XPS) or electron spectroscopy for chemical analysis (ESCA) and UV photoelectron spectroscopy (UPS), depending on the nature of the excitation source. Both have applications in examining the electronic structures and chemical bonding in biomolecules (8). The third historical path led to the study of surfaces by imaging the emitted photoelectrons. This work paralleled the development of electron lenses. As early as the 1930s, simple emission electron microscopes had been built to image photoelectrons emitted from metallic surfaces by UV light $(6,20)$.

Although photoelectron microscopy (PEM, or photoelectron emission

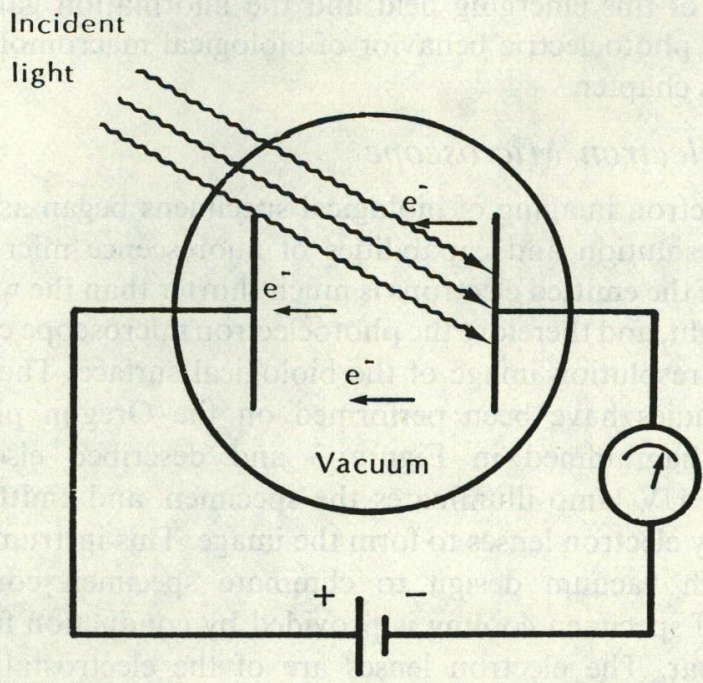

Figure 2 Diagram illustrating the essentials of the photoelectric effect. 
microscopy as it is called in Europe) was one of the earliest types of electron microscopy, it has not been a widely used technique. It has been developed to a limited extent in physics and metallurgical laboratories, primarily in Germany. An advance was made by Engel in the design of an instrument that used magnetic lenses (7). This design was incorporated into a commercial instrument, the Balzers KE-3. Approximately a dozen of these microscopes were produced in the early 1970 s for metallurgists. The availability of these instruments resulted in a period of increased activity and some interesting findings (29). With the increased awareness of the need for improved vacuum systems, the use of the early Balzers instrument has gradually declined. An ultrahigh vacuum instrument that combines the option of photoelectron investigations with other surface physics techniques has recently been introduced (2). For reviews and a bibliography of photoelectron microscopy applications in metallurgy and physics see References $(23,27,29)$.

In parallel with the development of photoelectron microscopy in the physical sciences in Germany, an independent effort, begun in 1969 and aimed at photoelectron imaging of biological specimens, is continuing in Oregon. An ultrahigh vacuum photoelectron microscope with electrostatic electron lenses was constructed for these studies. The first micrographs of organic and biological specimens were reported in 1972 (11). The photoelectron imaging of biological specimens has progressed steadily with improvements in instrumental design, knowledge of the photoelectric behavior of biological molecules, and experience in specimen preparation. An overview of this emerging field and the information gained to date regarding the photoelectric behavior of biological macromolecules is the subject of this chapter.

\section{The Photoelectron Microscope}

The photoelectron imaging of biological specimens began as an effort to extend the resolution and capabilities of fluorescence microscopy. The wavelength of the emitted electrons is much shorter than the wavelength of fluorescent light, and therefore the photoelectron microscope can provide a much higher resolution image of the biological surface. The majority of biological studies have been performed on the Oregon photoelectron microscope diagrammed in Figure 3 and described elsewhere (13). Light from a UV lamp illuminates the specimen, and emitted electrons are focused by electron lenses to form the image. This instrument is of oilfree, ultrahigh vacuum design to eliminate specimen contamination. The option of specimen cooling is provided by conduction from a liquid nitrogen dewar. The electron lenses are of the electrostatic unipotential type because they are easily accommodated in the ultrahigh vacuum 
design. The images are viewed with an image intensifier-TV system for selecting the specimen areas and focusing. The images are then recorded on photographic film by means of an internal camera system designed for compatibility with the ultrahigh vacuum microscope.

In order to maximize the solid angle of illumination the UV light is reflected from the mirrored anode onto the specimen in the photoelectron microscope, as diagrammed in Figure 3. The specimen is mounted on the cathode, and the emitted electrons are accelerated across the cathodeanode gap before entering the electron optical system. The optical system includes three lenses-an objective, intermediate, and projector-and is similar to that of a transmission electron microscope. In contrast to a TEM, however, there is no need for an electron gun because the specimen itself is the source of the electrons used to form the image. One can think of this instrument as a combination of the excitation source of a fluorescence microscope with the imaging system of a transmission electron microscope.

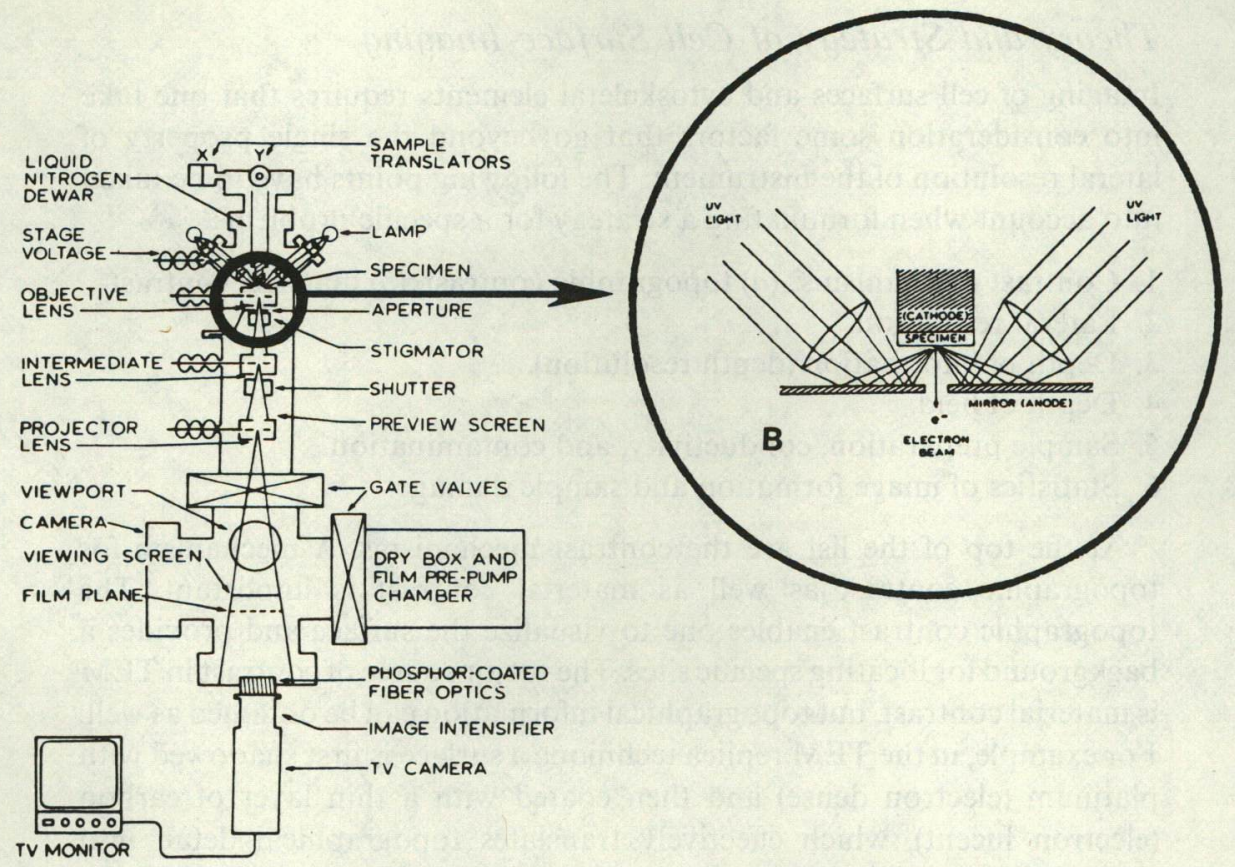

A

Figure $3(A)$ Schematic diagram of the Oregon photoelectron microscope, and $(B)$ an enlarged view of the UV optics that focuses light onto the specimen. Not shown: the cathode cup that surrounds the specimen except for a central 3- $\mathrm{mm}$ diameter aperture to allow UV light in and the electrons out. 
This is very different in concept, for example, from a scanning electron microscope, where a focused electron beam is used to scan the specimen and the image information is collected point by point sequentially. The analog to an SEM would be a system in which the specimen is scanned with a focused UV beam, and the information collected, one image element at a time. In this case the instrument would be limited to the resolution of the UV light spot size. This method of image formation is not used in PEM; rather, the entire portion of the specimen being viewed is illuminated with UV light, and the emitted electrons are focused simultaneously and continuously to form the image. Therefore, the resolution is limited only by the aberrations of the electron optics system and ultimately by the diffraction limit imposed by the wavelength of the emitted electrons. The specimen damage is also minimized by continuous illumination of the entire surface as opposed to subjecting the specimen to a high intensity spot of UV light, which would be needed to produce the same exposure in the scanning mode.

\section{Theory and Strategy of Cell Surface Imaging}

Imaging of cell surfaces and cytoskeletal elements requires that one take into consideration some factors that go beyond the single property of lateral resolution of the instrument. The following points have to be taken into account when formulating a strategy for a specific problem:

1. Contrast mechanisms: (a) topographic contrast, $(b)$ material contrast.

2. Lateral resolution.

3. Depth of information (depth resolution).

4. Depth of field.

5. Sample preparation, conductivity, and contamination.

6. Statistics of image formation and sample damage.

At the top of the list are the contrast mechanisms. A mechanism for topographic contrast as well as material contrast is important. The topographic contrast enables one to visualize the surface and provides a background for locating specific sites. The major source of contrast in TEM is material contrast, but topographical information can be obtained as well. For example, in the TEM replica technique a surface is first shadowed with platinum (electron dense) and then coated with a thin layer of carbon (electron lucent), which effectively translates topographical detail into material contrast (21). The replica does not provide any information as to material contrast inherent in the specimen itself (unless some of the specimen adheres to the replica, as is sometimes the case in colloidal gold labeling, for example). Topographical information in TEM can also be obtained by examining thin sections of specimens that have been stained 
with heavy metals. This provides a one-dimensional view of the cell surface but allows the imaging of the relationship of cytoplasmic elements to the cell surface. More information can be obtained by using serial sections, but the reconstruction process is laborious. This method is used primarily where the desired information can be obtained from one or a small number of sections, rather than a complete set of serial sections. It is valuable, for example, in studying cross sections of coated pits in receptor-mediated endocytosis $(1,15)$. In the TEM whole mount technique, the contrast available is primarily material contrast provided by heavy metal staining. As in the TEM replica method, there is no direct topographic contrast mechanism. The whole mount technique is used primarily in studies of cytosketelal elements and requires a high voltage TEM for increased penetration power through the thick specimens. Topographical contrast in SEM occurs because the secondary electron signal depends on the angle of incidence of the primary beam at the specimen surface and also on the angle of detection of the secondary electrons. Topographical contrast in SEM is enhanced by tilting the surface away from normal with respect to the probe beam. Material contrast based on atomic number differences can be observed in SEM through the use of a variety of detectors, making SEM more of an analytical microscope. The analytical capability is more useful in material science than in biological science applications. In biological specimens, except for metal ions, there is little variation in elemental composition and generally the specimens are coated, which suppresses some of the material contrast.

In PEM, both surface topography and differences in surface chemical composition produce contrast in the image. Material contrast is based on photoelectron yield, which depends on the valence electron levels of the surface materials, rather than on atomic number differences as in TEM and SEM. Material contrast will come up again in the discussion of photoelectron labeling. The origin of the topographical contrast in PEM is the effect of specimen surface relief on the electric field, which accelerates the low energy photoelectrons as they emerge from the surface $(24,27)$. The microfields at the surface of the specimen deflect the electrons, causing more of them to be intercepted by the aperture stop in the electron optical system. This is illustrated in Figure 4. An electron emitted from the top of a protrusion from the specimen surface (as, for example, the bump caused by nucleoli under the membrane) is undeflected as it leaves the specimen (ray 2). The two other electrons (rays 1 and 4) shown leaving the plane areas on either side of the protrusion are also undeflected. Each of these rays represents a narrow bundle of (parabolic) trajectories, which superimpose at the center of the aperture stop. However, ray 3 represents an electron leaving the sloping side of the protrusion. This ray is deflected by the electric 


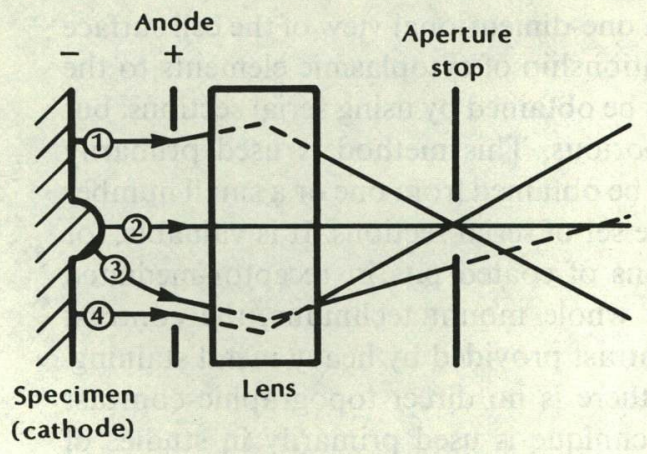

a

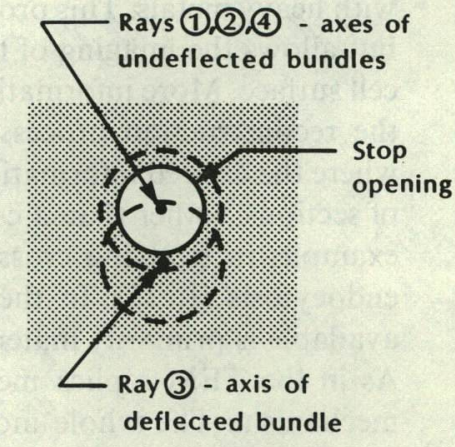

b

Figure 4 Diagram illustrating the origin of topographical contrast arising from the deflection of electrons emitted from sloping surfaces.

field, which causes the electron to pass through the aperture stop off-center. As a result, the trajectories surrounding ray 3 are intercepted by the aperture stop to a greater extent than are the trajectories surrounding rays 1,2 , and 4, as shown in the sketch in the right part of Figure 4. The net effect is that the images of the sides of a protrusion are darker than the top and surrounding flat areas. This effect enhances the contrast of small topographical features and makes them more readily visible in the photoelectron image.

Considering the origin of the topographic contrast in PEM, it is not surprising that if the topographic features are too rugged (e.g. rounded-up cells) the useful range will be exceeded, leading to loss of information from the image. On balance, the enhanced sensitivity of PEM to topographic features is of great value in viewing cell surface detail and cytoskeletal elements of cells, particularly where the specimens are well spread.

The second factor that must be taken into account is lateral resolution. Lateral resolution of all microscopes is ultimately limited by the Abbe diffraction equation (21)

$$
r=0.61 \lambda / \text { N.A. and N.A. }=n \sin \alpha
$$

where $r$ is the minimum distance between two object points for which the images can just be resolved, $\lambda$ is the wavelength of the radiation illuminating the object, N.A. is the numerical aperture, $n$ is the refractive index of the object space medium, and $\alpha$ is the half angle of acceptance of the objective lens. In all forms of electron microscopy the increase in resolution over that of the light microscope is due to the much shorter wavelength of the 
electrons. However, all electron microscopes have additional errors that are due to chromatic and spherical aberrations of the lenses, which prevent the resolution from reaching the diffraction limit calculated from the Abbe equation. The photoelectron microscope has additional aberrations inherent in the acceleration process. The way in which these aberrations arise can be seen by considering the motion of an electron in a uniform electric field. In a uniform accelerating field, the electrons travel along parabolic paths. The tangents to these paths at the end of the accelerating region are the incident rays for the electron optical system that follows. The tangents associated with a family of electron trajectories from a given specimen point form a virtual image of the specimen, which to first order is twice as far from the plane of tangency as is the specimen. The image distance is given by

$$
l^{*}=2 l /\left(1+\dot{z}_{\mathrm{o}} / \dot{z}_{\mathrm{a}}\right)
$$

where $l$ is the length of the accelerating space, $\dot{z}_{\mathrm{o}}$ is the axial component of the emission velocity, and $\dot{z}_{\mathrm{a}}$ is the axial component of the final velocity (24). The ratio $\dot{z}_{\mathrm{o}} / \dot{z}_{\mathrm{a}}$ is very small, and the image distance can be expressed as follows in terms of the emission energy and angle and the accelerating voltage:

$$
l^{*}=2 l\left(1-\sqrt{V_{\mathrm{o}} / V_{\mathrm{a}}} \cos \alpha\right)
$$

where $V_{\mathrm{o}}$ and $V_{\mathrm{a}}$ are the beam voltages before and after acceleration, and $\alpha_{\mathrm{o}}$ is the angle of emission measured from the normal to the surface (the beam voltage is defined by $e V=m v^{2} / 2$ ). Equation 5 shows that the virtual image contains both spherical and chromatic aberration (of the undercorrected type), as evidenced by the dependence of the distance $l^{*}$ on both the emission angle and the emission energy. This image, after action by the aperture lens effect at the termination of the acceleration field, becomes the object for the objective lens, and the aberrations are combined in a coherent manner with the longitudinal aberrations produced by the objective lens. As a consequence, the geometrical aberrations in the photoelectron microscope are larger than those due to the lenses alone, and the optimum resolution (achieved by a compromise with the diffraction error) is not as good as for a transmission microscope. For example, our PEM objective lens was tested to better than $20 \AA$ in a TEM, whereas the theoretical optimum resolution of the existing PEM is about $50 \AA$. At the present time, the Oregon PEM resolution is about $100-150 \AA$. The difference is due to a larger than optimum angular aperture currently in use to allow a larger beam current. With the installation of an improved UV-optical system the aperture will be reduced to the optimum value of about $30 \mu \mathrm{m}$.

Since many integral membrane proteins are on the order of $50 \AA$ in diameter, this photoelectron microscope has the potential of imaging 
membranes to single protein resolution. Looking further into the future, there is the possibility of using corrected electron optics, in analogy with the use of compound lenses in optical microscopy. This would involve more complex optical systems, for example lenses in combination with mirrors, to reduce the aberrations and approach the diffraction limit. The diffraction limit for a $1-\mathrm{eV}$ electron is on the order of $10 \AA$. A second reason for considering corrected electron optics is to utilize larger apertures to collect and image more of the electrons emitted from the slopes of topographical features. This would increase the range of specimen topography that could be imaged at high resolution.

The third factor to be considered, the depth of information, is of particular interest when imaging surfaces and should be considered in any microscopy experiment. The depth of information is defined as the distance below the surface from which information is contributed at a specified resolution (18). The depth of information in the TEM replica technique is essentially zero, since the replica records only surface information. The depth of information in TEM of thin sections is on the order of the section thickness, and for whole mount studies it more nearly corresponds to the entire specimen thickness. The depth of information in PEM is extremely short because the escape depth of the electrons is very small. The UV light penetrates deeply into biological specimens, but only those electrons photoionized at or very near the surface can escape and contribute to the image. The majority of electrons that escape have originated in a layer equal to or less than the thickness of the plasma membrane (18). In SEM the depth of information is small enough to be quite useful but is greater than that in PEM (5). Information from beneath and around the probe beam is merged into the signal. Even in the secondary electron mode, the depth of information depends on the depth from which a significant number of backscattered electrons contribute secondaries to the signal, and this depth is greater than the escape depth for secondary electrons or, in PEM, for photoelectrons.

The fourth factor, the depth of field, is the range in depth in the object that appears to be in focus. The depth of field $(D F)$ in all microscopes is inversely proportional to the product of the magnification $M$ times the half angle of acceptance $\alpha$ of the objective lens. TEM and SEM have the same depth of field $(D F=2 b / \alpha$, where $b=0.2 \mathrm{~mm} / M$ and $M$ is within the meaningful as opposed to the empty magnification range). SEM micrographs often given the impression of a greater depth of field than TEM micrographs, because the TEM micrographs are usually recorded at greater magnification. For PEM the expression is not very different, $D F=3 b / 2 \alpha$, providing that two planar regions at different depths are being brought into focus. However, in regions of significant topographical relief the working depth of field in 
PEM is greatly reduced because of the electron optical effect of the microfields (25). In practice, the depth of field is not usually a limiting factor. In all of these techniques the depth of field is adequate for a wide range of cell surface and cytoskeletal studies.

The remaining two factors that need to be considered involve properties of the specimen as well as the imaging technique. In general the cell must be fixed and dried, or frozen, whether it is to be replicated or observed directly in an electron microscope. In SEM the dehydrated or frozen specimen is usually, although not always, coated with a thin conductive metal or carbon layer, and in the TEM serial section technique the specimen is embedded and stained. In PEM the cells are normally grown on conductive glass discs and are fixed, dehydrated, and observed directly without staining or metal coating. It is important in all of these surface studies that specimen contamination be minimized or avoided because of the short depth of information.

Sample damage, and not resolution, is often the limiting factor in electron microscopy studies of biological specimens. The question is not whether specimen damage occurs in all forms of microscopy, but whether a sufficient number of information-carrying electrons can be recorded per picture element (pixel) before the specimen structure changes in some significant way at the resolution of interest. Specimen damage in TEM and SEM results eventually in etching and mass loss by the incident electron beam. In PEM, instead of a beam of electrons, UV light is focused on the specimen. In the specimens examined thus far, the UV light has not produced any noticeable alterations in the topography of the specimens, although some photochemical reactions occur as evidenced by an increased brightness of the specimens with time (9). UV light also causes changes in image brightness in fluorescence microscopy, for example the well-known bleaching of fluorescent labels. The brightening effect in PEM is used to advantage in permitting higher magnification images by waiting until the image brightens, just as in fluorescence microscopy advantage is taken of fluorescence bleaching of dye molecules by the UV light in certain experiments, for example measurements of lateral diffusion.

The question of the extent of specimen damage is closely related to the statistics of image formation. The signal-to-noise ratio $(S N R)$ is given by the formula $(17,28)$

$$
S N R=\left(n_{2}-n_{1}\right) /\left(n_{2}+n_{1}\right)^{1 / 2}
$$

where $n_{2}$ is the number of electrons emitted from a resolution element containing the signal (for example a labeled receptor site), and $n_{1}$ is the number of electrons emitted from a resolution element containing background proteins and lipids. An $S N R$ of 6 will produce a satisfactory 
image (28), and Equation 4 provides some insight as to how many electrons must be emitted in order to distinguish an object by material contrast from the background noise. For example, for a single emitting label in a 5-nm resolution element with $n_{2}=30 n_{1}$, an $S N R$ of 6 would require, from Equation 6, emission of about 40 electrons from the resolution element containing the signal. It has been shown from beam current measurements as a function of time that the aromatic carcinogen benzo(a)pyrene can emit electrons a large number of times (17). After each electron is emitted, the molecule recaptures an electron from the cathode and the process is repeated. This provides another indication that the damage rate is relatively low, at least for aromatic molecules.

\section{Photoelectron Images of Eucaryotic Cells}

All organic molecules will photoemit electrons when subjected to UV light of sufficient energy, and therefore it is not surprising that all cells examined thus far have produced photoelectron images. The image quality varies with the type of cell, and the most readily viewed cells are those that are well-spread. A recent review of the earlier work on photoelectron imaging of tissue culture cells, sperm, thin sections, photosynthetic bacteria, viruses, nucleic acids, and other biological specimens is presented elsewhere (12). Here we provide a few examples from current work to illustrate the quality of micrographs being obtained.

An example of cell surface imaging is shown in Figure 5. This photoelectron micrograph is of two guinea pig sperm that happened to overlap. One sperm is intact and the other one has lost its acrosome. The main features of a sperm cell including the acrosome, nucleus, connecting piece between the head and tail, the perforatorium, plasma membrane, and inner acrosomal membrane (exposed after loss of the acrosome) are readily identified in photoelectron micrographs (R. J. Mrsny and O. H. Griffith, unpublished). Although membranes in this preparation appeared intact, the air drying of this sperm as a final preparative step probably accounts for the apparent tears in the plasma membrane seen in the region of the acrosome at the periphery of the nucleus-the region separating the proximal and distal segments of the acrosome. Photoelectron micrographs of a variety of other cell types have been obtained including cultured human fibroblasts, endothelial cells, epithelial cells, and tumor-derived cells (12).

A comparison of photoelectron and immunofluorescence images of the same cells has recently been made (22). This type of study is illustrated in Figure 6, using RAT-1 fibroblast cells prepared for the visualization of actin. The immunofluorescence micrograph (A) shows the typical pattern of actin-containing stress fibers of these cells. The photoelectron micrograph (B) is of the same specimen area at a comparable magnification, and (C, D) 
are successive enlargements. The photoelectron micrographs are remarkably similar to the immunofluorescence micrograph, except that more detail is seen. In the photoelectron micrographs, it is possible to see the damage to the cell surface caused by the permeabilization treatment used to permit access of the antibodies to the cell interior. The presence of the fluorescent dye is not detected in photoelectron micrographs at the labeling levels used here (i.e. 1-2 dyes/protein). The main contrast mechanism is topographical, so that all internal structures exposed by the permeabilization step, and not just actin-containing stress fibers, are detected. The stress fibers stand out because of their dimensions, which are very likely enhanced somewhat by the two layers of antibody. These micrographs show that PEM can be used to image not only cell surfaces but also internal

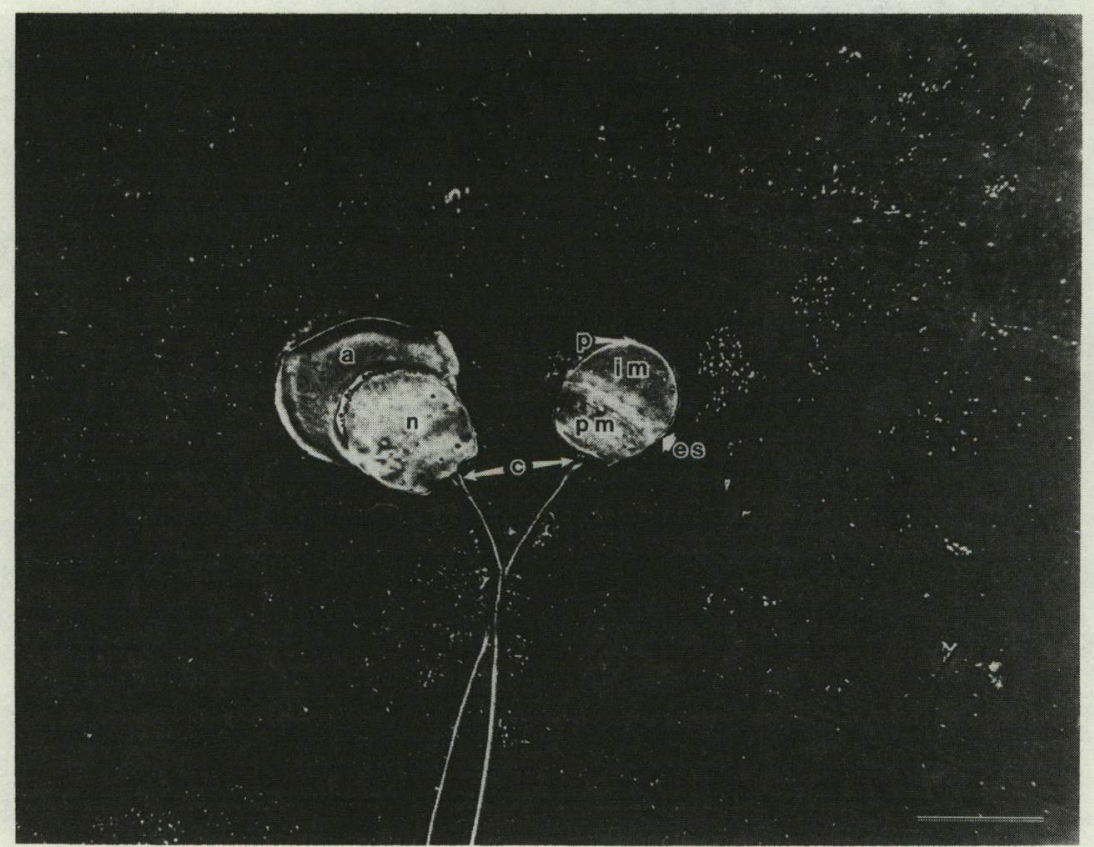

Figure 5 Photoelectron micrograph of washed guinea pig epididymal sperm. The sperm were fixed in paraformaldehyde as described in Reference 26. A droplet of washed sperm was placed on a tin-oxide coated, Alcian-blue treated glass coverslip, and excess suspension was withdrawn with filter paper, followed by several water washes and air drying. The most prominent features are indicated : $a$, acrosomal region; $n$, nuclear region; $c$, connecting piece; $p$, perforatorium; es, equatorial segment; $p m$, plasma membrane; and im, inner acrosomal membrane. The sperm on the right has lost the acrosome (the large structure still present in the sperm on the left). Bar $=5 \mu \mathrm{m}$. 

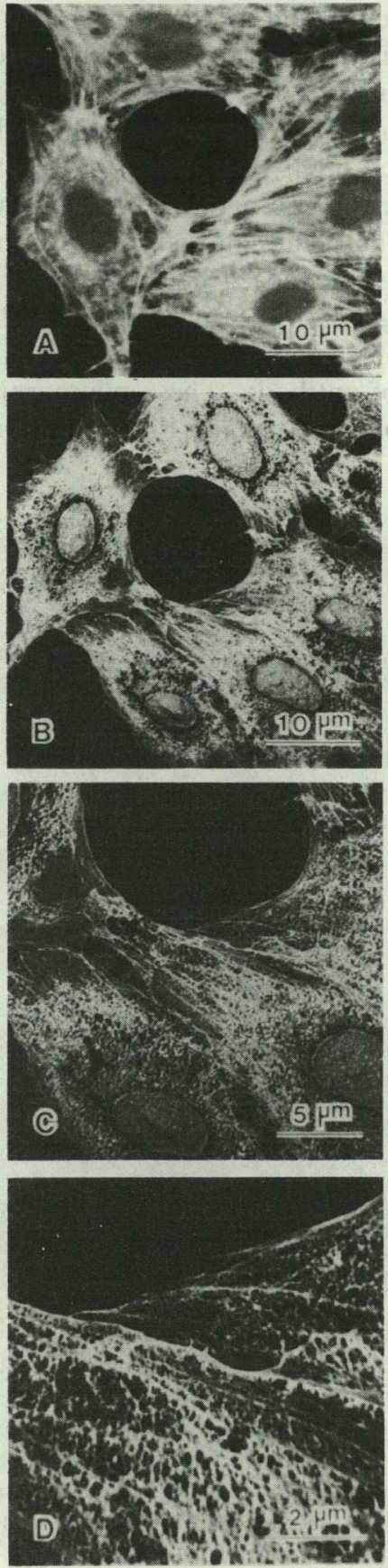

Figure 6 Immunofluorescence micrograph $(A)$ and photoelectron micrographs at increasingly higher magnifications $(B-D)$ of the same Rat-1 fibroblast prepared for the visualization of actin as described in Reference 22. 
cellular structures, provided that they are sufficiently exposed during specimen preparation.

\section{Photoelectron Labeling}

One of the most useful applications of photoelectron imaging is in the extension of immunofluorescence microscopy to cell biological studies requiring higher resolution. This methodology requires, in addition to the photoelectron microscope, the development of photoelectron markers to make specific sites visible. The experiment is illustrated in the diagram of Figure 7. A marker is coupled either by a covalent bond or by high affinity adsorption to an antibody or other site-specific ligand. The cell preparation is then exposed to the purified marker-antibody complex and is washed if necessary to minimize nonspecific binding. The photoelectron image now contains, in addition to a topographical map of the specimen, information regarding the identity and antigenicity of the exposed structures. By analogy with immunofluorescence microscopy, this technique is called immunophotoelectron microscopy.

Only in recent years has knowledge of the photoelectric behavior of biochemicals and potential markers progressed to the point where photoelectron labeling is becoming practical. The properties of an ideal marker are listed in Figure 7. The first attempts involved fluorescence dyes, which in fact are photoemissive. However, the overall increased photoemis-

\section{CEL SUPFACE LABE ING}

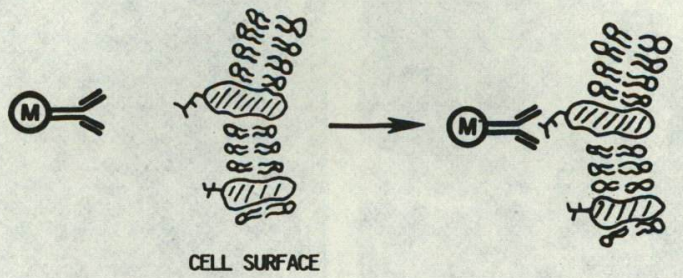

REQUIRENENTS FOR PEN MARKERS (M)
(A) High Photoelectron Cuantum Yield
(B) DistinCTIVE SIZE AND SHAPE
(C) STABLE
(D) High Affinity Bimoing to Ligand
(E.6. ANTIBOOY, LECTIN, BIOTIN)
(E) Mimimal Nonspecific Binding to CELL SURfaces

Figure 7 Diagram illustrating the concept of photoelectron labeling. 
sion of the normal cell components, with exposure to UV light, had the effect of decreasing the expected contrast (10). There are possible uses of dyes, as well as techniques yet to be tried (e.g. triplet state probes). However, more promising at present is the colloidal gold technology that is being developed for use in TEM and SEM (16). Small highly uniform gold spheres can be obtained in a range of diameters (16), and under the proper conditions the particles bind strongly and irreversibly to antibodies and plant lectins. Recently, it has been shown that colloidal gold provides sufficient enhanced photoemission from labeled cellular components to make them visible in the photoelectron microscope $(3,4)$. An example of an immunophotoelectron microscopy experiment using colloidal gold is shown in Figure 8 . Here microtubules ( $25 \mathrm{~nm}$ in diameter) have been labeled with both a fluorescent dye and colloidal gold in a three-step antibody procedure to permit a direct comparison of immunofluorescence microscopy (Figure 8A) and immunophotoelectron microscopy (Figure
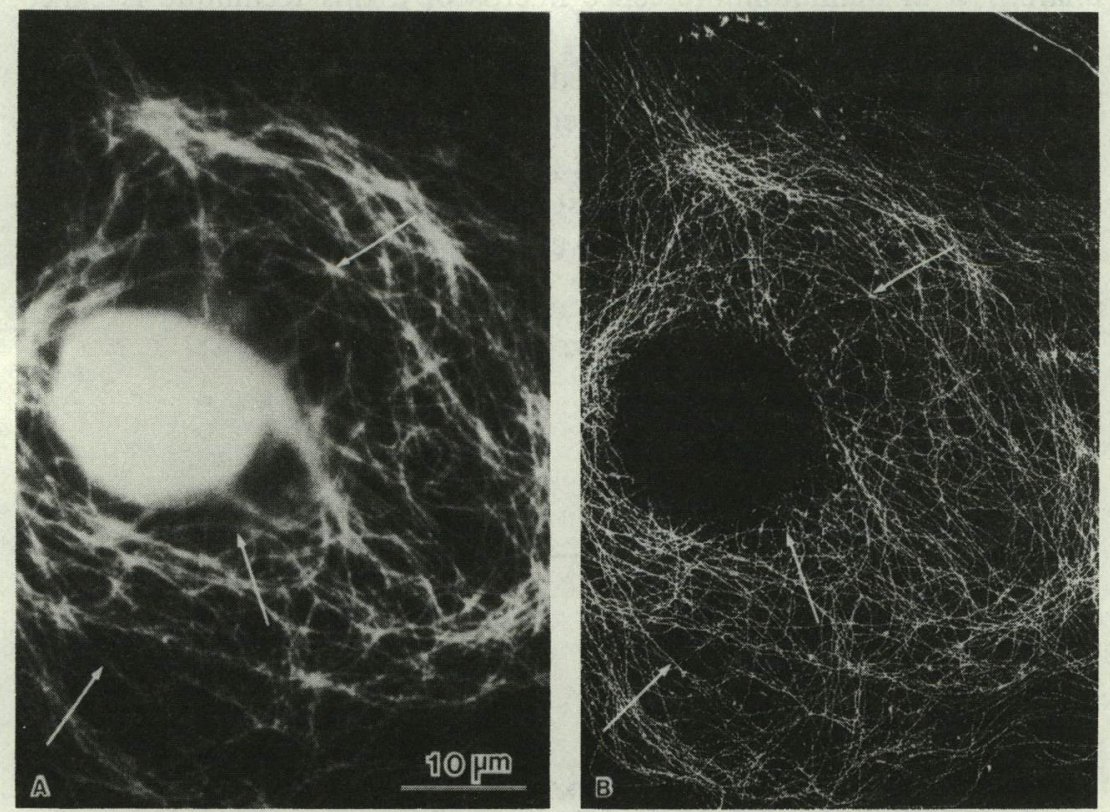

Figure 8 Immunofluorescence micrograph $(A)$ and immunophotoelectron micrograph $(B)$ of the same CV-1 epithelial cell following detergent extraction under conditions that preserve microtubules (3). A three-step, double-labeling procedure was used to visualize the microtubule network. The first antibody recognized the microtubules, the second carried a fluorescent label (rhodamine), and a third was bound to a photoelectron marker (20-nm colloidal gold). After the fluorescence micrograph was taken, the specimen was glutaraldehyde-fixed and critical point dried for photoelectron microscopy. Some of the numerous microtubules that can be identified in both the fluorescence and photoelectron micrographs are identified by arrows. 
8B). The network of microtubules is visible in both micrographs. Individual gold particles are clearly seen in Figure $8 \mathrm{~B}$ because of their enhanced emission and topography. Immunophotoelectron microscopy has also been performed successfully using a two-step procedure to minimize the increased diameter caused by the presence of the antibodies. The labeled microtubules stand out against the background of other cellular components (3).

\section{Conclusions}

Photoelectron imaging is the electron optical analog of fluorescence microscopy. It shares with fluorescence the advantage that small objects are often easier to detect by their emitted signals than by absorption or scattering (the same principle applies to viewing stars or city lights at night).

Photoelectron images can now be obtained of cell surfaces and cytoskeletal elements, and photoemissive markers coupled to antibodies can be used to elucidate the distribution of specific proteins and to correlate structure and function. This approach, immunophotoelectron microscopy, has the potential of extending immunofluorescence microscopy to much higher resolution studies of biological surfaces. Photoelectron imaging is not, of course, a cure-all. One limitation is that, like most forms of electron microscopy, the specimen must be either dehydrated or quick frozen before viewing. Another limitation is imposed by the great sensitivity to specimen topography: although this topographic contrast is an advantage in photoelectron imaging of fine surface detail, specimens with pronounced topography may exceed the useful range of this technique. However, mammalian sperm and many cultured cell types fall within this range and can be studied by photoelectron imaging. The current resolution is 100 to $150 \AA$ with a design goal for the present instrument of $50 \AA$. There is a longterm possibility of $10-\AA$ resolution and a greater range of specimen topographies with corrected electron optics (and luck!). Thus, single protein resolution (e.g. $50 \AA$ ) is within reach. However, even at the present resolution, the high sensitivity to surface detail, the short depth of information, and the ability to image uncoated and unstained specimens make photoelectron imaging a promising approach in studies of cell surface receptors, cytoskeletal elements, and other cellular components.

\section{ACKNOWLEDGMENTS}

We are indebted to Douglas L. Habliston for obtaining the photoelectron micrographs presented here. We wish to thank Drs. Karen K. Nadakavukaren, G. Bruce Birrell, and Randall T. Mrsny for useful discussions. This work was supported by Grant No. CA11695 from the National Cancer Institute. 


\section{Literature Cited}

1. Anderson, R. G. W., Goldstein, J. L., Brown, M. S. 1977. Nature 270:695

2. Bethge, H., Klana, M. 1982. Ultramicroscopy 11:207

3. Birrell, G. B., Habliston, D. L., Nadakavukaren, K. K., Griffith, O. H. 1984. Proc. Natl. Acad. Sci. USA. In press

4. Birrell, G. B., Rose, S. M., Griffith, O. H. 1983. Ultramicroscopy $12: 213$

5. Bode, M., Pfefferkorn, G., Schur, K., Wegmann, L. 1972. J. Microsc. 95:323

6. Brüche, E. 1933. Z. Phys. 86:448

7. Engel, W. 1968. Proc. 6th Int. Congr. Electron Microsc., Kyoto, p. 217

8. Ghosh, P. K. 1983. Introduction to Photoelectron Spectroscopy. New York: Wiley

9. Griffith, O. H., Holmbo, D. L., Habliston, D. L., Nadakavukaren, K. K. 1981. Ultramicroscopy $6: 149$

10. Griffith, O. H., Houle, W. A., Kongslie, K. F., Sukow, W. W. 1984. Ultramicroscopy 12:299

11. Griffith, O. H., Lesch, G. H., Rempfer, G. F., Birrell, G. B., Burke, C. A., et al. 1972. Proc. Natl. Acad. Sci. USA 69:561

12. Griffith O. H., Nadakavukaren, K. K., Jost, P. C. 1984. Scanning Electron Microsc. 2: 633

13. Griffith, O. H., Rempfer, G. F., Lesch, G. H. 1981. Scanning Electron Microsc. 2: 123

14. Griffith, O. H., Rempfer, G. F., Nadakavukaren, K. K. 1982. Proc. 10th Int. Congr. Electron Microsc., Hamburg 1:59

15. Handley, D. A., Arbeeny, C. M., Witte, L. D., Chien, S. 1981. Proc. Natl. Acad. Sci. USA $78: 368$
16. Horisberger, M. 1981. Scanning Electron Microsc. 2:9

17. Houle, W. A., Brown, H. M., Griffith, O. H. 1979. Proc. Natl. Acad. Sci. USA $76: 4180$

18. Houle, W. A., Engel, W., Willig, F., Rempfer, G. F., Griffith, O. H. 1982. Ultramicroscopy $5: 371$

19. Hughes, A. L., Du Bridge, L. A. 1932. In Photoelectric Phenomena, pp. 7-37. New York: McGraw-Hill

20. Mahl, H., Pohl, J. 1935. Z. Tech. Phys. $16: 219$

21. Meek, G. A. 1976. Practical Electron Microscopy for Biologists. New York: Wiley

22. Nadakavukaren, K. K., Chen, L. B., Habliston, D. L., Griffith, O. H. 1983. Proc. Natl. Acad. Sci. USA 80: 4012

23. Pfefferkorn, G., Schur, K., eds. 1979. Proc. 1st Int. Conf. Emission Electron Microsc., Tübingen. Beitr. elektronenmikroskop. Direktabb. Oberfl. 12/2, pp. 1-234

24. Rempfer, G. F., Nadakavukaren, K. K., Griffith, O. H. 1980. Ultramicroscopy $5: 437$

25. Rempfer, G. F., Nadakavukaren, K. K., Griffith, O. H. 1980. Ultramicroscopy $5: 449$

26. Schwartz, M. A., Koehler, J. K. 1979. Biol. Reprod. 21:1295

27. Schwarzer, R. 1981. Microsc. Acta 84: 51

28. Van Dorsten, A. C. 1960. In Proc. Eur. Rég. Conf. on Electron Microsc., ed. A. Houwink, B. Spit, 1:64-68. Delft, Netherlands

29. Wegmann, L. 1972. J. Microsc. 96:1 